\title{
Labeling trees with a condition at distance two
}

\author{
Tiziana Calamoneri * Andrzej Pelc ${ }^{\dagger} \quad$ Rossella Petreschi *
}

\begin{abstract}
An $L(h, k)$-labeling of a graph $G$ is an integer labeling of vertices of $G$, such that adjacent vertices have labels which differ by at least $h$, and vertices at distance two have labels which differ by at least $k$. The span of an $L(h, k)$-labeling is the difference between the largest and the smallest label. We investigate $L(h, k)$-labelings of trees of maximum degree $\Delta$, seeking those with small span. Given $\Delta, h$ and $k$, span $\lambda$ is optimal for the class of trees of maximum degree $\Delta$, if $\lambda$ is the smallest integer such that every tree of maximum degree $\Delta$ has an $L(h, k)$-labeling with span at most $\lambda$. For all parameters $\Delta, h, k$, such that $h<k$, we construct $L(h, k)$-labelings with optimal span. We also establish optimal span of $L(h, k)$-labelings for stars of arbitrary degree and all values of $h$ and $k$.
\end{abstract}

Key words: tree, vertex labeling, $L(h, k)$-labeling, maximum degree

\footnotetext{
*Dipartimento di Informatica, Università degli Studi di Roma "La Sapienza", 00198 Roma, Italy. E-mail: \{calamo,petreschi\}@dsi.uniroma1.it

${ }^{\dagger}$ Département d'informatique, Université du Québec en Outaouais, Gatineau, Québec J8X 3X7, Canada. E-mail: pelc@uqo.ca

Supported in part by NSERC grant OGP 0008136 and by the Research Chair in Distributed Computing of the Université du Québec en Outaouais . This research was done during the visit of Andrzej Pelc, supported by a visiting fellowship from the Università degli Studi di Roma "La Sapienza".
} 


\section{Introduction}

In classic vertex coloring of graphs (cf. [18]), a condition is imposed only on colors of adjacent nodes: a proper coloring can be viewed as an integer labeling of vertices, such that adjacent vertices have labels differing by at least 1 . Recently, vertex labelings of graphs respecting a stronger condition were intensly studied $[1,8,9,15,20,22]$ : restrictions are imposed both on labels of adjacent nodes and of nodes at distance 2 in the graph. An $L(h, k)$-labeling of a graph $G$ is an integer labeling of vertices of $G$, such that adjacent vertices have labels which differ by at least $h$, and vertices at distance two have labels which differ by at least $k$. A span of such a labeling is the difference between the largest and the smallest label. Hence a classic vertex coloring of graphs is an $L(1,0)$ labeling, and the smallest span of such a labeling for a given graph $G$ is $\chi(G)-1$, where $\chi$ is the chromatic number.

$L(2,1)$-labelings were first studied in [15] in connection with the channel assignment problem (cf. [16]), in which close transmitters (vertices at distance 2) have to be assigned different channels, and very close transmitters (adjacent vertices) have to be assigned channels at least two apart. In many subsequent papers, e.g., [2, 3, 5, 6, 7, 9, 11, 19, 21, 23] the minimum span of $L(2,1)$-labelings was studied for different classes of graphs. In [10], the authors introduced the general notion of $L(h, k)$ labelings of graphs as a special case of the notion of $L\left(m_{1}, \ldots m_{N}\right)$-labelings introduced in [15]. This notion was further studied in $[4,12,13,14,17]$. In particular, in [13], the authors investigate $L(h, k)$-labelings of trees for $h \geq k$ and $\Delta \geq 3$. For these parameters they obtain optimal span for infinite trees. In this paper, we present results that are complementary with respect to those in [13]. ${ }^{*}$ Namely, we investigate $L(h, k)$-labelings of trees, for arbitrary positive integers $h<k$, seeking such labelings with small span. Unlike for classic vertex coloring, the smallest span of an $L(h, k)$ labeling of trees heavily depends on their maximum degree $\Delta$, hence we use it as a parameter in our considerations. We look at the problem from a different point of view with respect to [9], where the authors designed a polynomial algorithm to find the minimum span of an $L(2,1)$-labeling for a given tree. Instead, we look at the class of trees of maximum degree $\Delta$, as a whole. Given $\Delta, h$ and $k$, span $\lambda$ is optimal for the class of trees of maximum degree $\Delta$, if $\lambda$ is the smallest integer such that every tree of maximum degree $\Delta$ has an $L(h, k)$-labeling with span at most $\lambda$. For all parameters $\Delta, h, k$, such that $h<k$, we construct $L(h, k)$-labelings with optimal span. For $h \geq k$, values of optimal span follow from [13].

The paper is organized as follows. In Section 2, we introduce terminology and summarize our results. Section 3 is devoted to the derivation of optimal span for the class of trees of maximum

${ }^{*}$ At the time of writing this paper, we were unaware of [13] which was not published yet. Our approach and techniques significantly differ from those in [13]. 
degree $\Delta$, when $h<k$. In Section 4, we derive the value of the optimal span for stars of arbitrary degree, for all values of $h$ and $k$. Finally, in Section 5, we conclude the paper presenting open problems.

\section{Terminology and summary of results}

\subsection{Terminology}

Given positive integers $h$ and $k$, an $L(h, k)$-labeling of a graph $G=(V, E)$ is a function $L: V \longrightarrow \mathcal{N}$ (where $\mathcal{N}$ is the set of natural numbers) such that $|L(u)-L(v)| \geq h$, if $u, v$ are adjacent, and $|L(u)-L(v)| \geq k$, if $u$ and $v$ are at distance 2. For an $L(h, k)$-labeling $L$, the integer $L(v)$ is called the label of $v$. The span of an $L(h, k)$-labeling is the difference between the largest and the smallest value of $L$. Without loss of generality we assume that the smallest value of $L$ is 0 .

$\Delta \geq 2$ denotes the maximum degree of a tree. For any $\Delta \geq 2$ and for any positive integers $h$ and $k$, we denote by $\lambda(\Delta, h, k)$ the smallest integer $\lambda$ such that every tree of maximum degree $\Delta$ has an $L(h, k)$-labeling with span at most $\lambda$.

We often consider trees as rooted at a fixed vertex. In this case, usual notions of parent, child and level are meant with respect to this root. Level $i$ is the set of vertices at distance $i$ from the root. The height of a tree is the largest index of its level.

\subsection{Summary of results}

We derive the following exact values of $\lambda(\Delta, h, k)$, for $h<k$ :

- if $h \leq k / 2$ then $\lambda(\Delta, h, k)=h+(\Delta-1) k$;

- if $k / 2 \leq h \leq \frac{\Delta}{2 \Delta-1} k$ then $\lambda(\Delta, h, k)=(2 \Delta-1) h$;

- if $\frac{\Delta}{2 \Delta-1} k \leq h \leq k$ then $\lambda(\Delta, h, k)=\Delta k$;

The detailed proofs of these results are presented in the next section. In all cases, upper bounds on $\lambda(\Delta, h, k)$ are proved by constructing an $L(h, k)$-labeling for all complete trees of degree $\Delta$, i.e., for trees in which all internal vertices have degree $\Delta$. Lower bounds on $\lambda(\Delta, h, k)$ are proved by constructing a tree of maximum degree $\Delta$, for which every $L(h, k)$-labeling has span at least equal to some given integer. More precisely, we show that the complete tree of degree $\Delta$ and sufficiently large height must have this property.

As we will show, relatively large values of $\lambda(\Delta, h, k)$ are witnessed by trees of large height. This fact is not accidental: we show that for trees of height 1, i.e., for stars, the span of $L(h, k)$-labelings is 
in fact smaller. Indeed, for stars of degree $\Delta$, we establish the minimum span of an $L(h, k)$-labeling for arbitrary $h$ and $k$. Its value is:

- $(\Delta-1) k$, if $h \leq k / 2$;

- $(\Delta-2) k+2 h$, if $k / 2 \leq h \leq k$;

- $(\Delta-1) k+h$, if $h \geq k$.

\section{Derivation of $\lambda(\Delta, h, k)$, for $h<k$}

In this section we derive exact values of $\lambda(\Delta, h, k)$, for $h<k$.

Theorem 3.1 If $h \leq k / 2$ then $\lambda(\Delta, h, k)=h+(\Delta-1) k$.

Proof: In order to prove $\lambda(\Delta, h, k) \leq h+(\Delta-1) k$, consider any complete tree of degree $\Delta$, rooted at node $r$. Consider the following labeling $L . L(r)=0$, and labels of all other nodes are defined as follows. Labels of nodes at even levels are taken from the set $A=\{0, k, 2 k, \ldots,(\Delta-1) k\}$, and labels of nodes at odd levels are taken from the set $B=\{h, h+k, h+2 k, \ldots, h+(\Delta-1) k\}$. Children of $r$ get all labels from $B$. For a given internal node $v \neq r$ at an even (resp. odd) level, children of $v$ get all labels from the set $B$ (resp. $A$ ), except the label of the parent of $v$. Since $h \leq k / 2$, the above defined labeling is an $L(h, k)$-labeling. Its span is $h+(\Delta-1) k$.

In order to prove $\lambda(\Delta, h, k) \geq h+(\Delta-1) k$, consider a complete tree $T$ of degree $\Delta$ and height $h+(\Delta-1) k$. Let $r$ denote its root. Suppose that there exists an $L(h, k)$-labeling $L$ of $T$ with span strictly smaller than $h+(\Delta-1) k$. We have $L(r)<h+(\Delta-1) k$.

Claim. There exists an internal vertex $v$ of $T$ such that labels of all neighbors of $v$ are larger than $L(v)$.

We construct the following sequence $\left(v_{i}: i<h+(\Delta-1) k\right)$ of vertices. $v_{0}=r$. If $v_{i}$ satisfies the claim, we are done. Otherwise, $v_{i+1}$ is any neighbor of $v_{i}$ such that $L\left(v_{i+1}\right)<L\left(v_{i}\right)$. Since $L(r)<h+(\Delta-1) k$, there cannot exist a descending sequence of $h+(\Delta-1) k+1$ non-negative integers starting from $L(r)$. Hence some $v_{i}$, for $i<h+(\Delta-1) k$ must satisfy the claim.

Now the proof of the theorem can be concluded as follows. Let $v$ be an internal vertex of $T$ satisfying the claim. Labels of all $\Delta$ neighbors of $v$ must be at least $h$, and differences between any pair of them must be at least $k$. Hence the largest label of a neighbor of $v$ must be at least $h+(\Delta-1) k$. This is a contradiction. 
Theorem 3.2 If $k / 2 \leq h \leq \frac{\Delta}{2 \Delta-1} k$ then $\lambda(\Delta, h, k)=(2 \Delta-1) h$.

Proof: In order to prove $\lambda(\Delta, h, k) \leq(2 \Delta-1) h$, consider any complete tree of degree $\Delta$, rooted at node $r$. Consider the following labeling $L . L(r)=0$, and labels of all other nodes are defined as follows. Labels of nodes at even levels are taken from the set $A=\{0,2 h, 4 h, \ldots,(2 \Delta-2) h\}$, and labels of nodes at odd levels are taken from the set $B=\{h, 3 h, 5 h, \ldots,(2 \Delta-1) h\}$. Children of $r$ get all labels from $B$. For a given internal node $v \neq r$ at an even (resp. odd) level, children of $v$ get all labels from the set $B$ (resp. $A$ ), except the label of the parent of $v$. Since $h \geq k / 2$, the above defined labeling is an $L(h, k)$-labeling. Its span is $(2 \Delta-1) h$.

In order to prove $\lambda(\Delta, h, k) \geq(2 \Delta-1) h$, consider a complete tree $T$ of degree $\Delta$ and height $(2 \Delta-1) h+2 \Delta-1$. Let $r$ denote its root. Suppose that there exists an $L(h, k)$-labeling $L$ of $T$ with span strictly smaller than $(2 \Delta-1) h$. We have $L(r)<(2 \Delta-1) h$. The following claim is proved similarly as that in the proof of Theorem 3.1.

Claim 1. There exists a vertex $v$ of $T$ at level at most $(2 \Delta-1) h$ such that labels of all neighbors of $v$ are larger than $L(v)$.

Let $v$ be a node satisfying the claim. Differences between labels of all neighbors of $v$ must be at least $k$. If $L(v) \geq k$ then $a^{\prime} \geq \Delta k \geq(2 \Delta-1) h$, where $a^{\prime}$ is the largest label of any neighbor of $v$. This is a contradiction. Hence $L(v)<k$.

From now on, consider the tree $T$ as rooted in $v$ (not in $r$ ). Notions of child, sibling and parent are now meant with respect to root $v$. Let $w$ be the child of $v$ with smallest label. Denote $a=L(w)$. Hence $a \geq h$. If $a \geq k$ then $a^{\prime} \geq \Delta k \geq(2 \Delta-1) h$, where $a^{\prime}$ is the largest label of any child of $v$. This is a contradiction. Hence $a<k$.

We construct two sequences of vertices: $\left(x_{1}, x_{2}, \ldots, x_{\Delta-1}\right)$ and $\left(y_{1}, y_{2}, \ldots, y_{\Delta-1}\right)$. The construction is inductive. $x_{1}$ is the child of $w$ with smallest label, and $y_{1}$ is the child of $x_{1}$ with smallest label. Suppose that $x_{1}, x_{2}, \ldots, x_{r-1}$ and $y_{1}, y_{2}, \ldots, y_{r-1}$ are already constructed. $x_{r}$ is the child of $y_{r-1}$ with the $r$ th label in increasing order, and $y_{r}$ is the child of $x_{r}$ with the $r$ th label in increasing order. Since $v$ is at level at most $(2 \Delta-1) h$ and $T$ has height $(2 \Delta-1) h+2 \Delta-1$, the above construction can be carried out.

\section{Claim 2.}

1. $2 r h \leq L\left(x_{r}\right)<r k+h$.

2. $(2 r+1) h \leq L\left(y_{r}\right)<(r+1) k$. 
Claim 2 is proved by induction on $r$. We first prove it for $r=1$.

Suppose that $L\left(x_{1}\right)<a$. Then $L\left(x_{1}\right)<k$. We also have $L(v)<k$ and $\left|L(v)-L\left(x_{1}\right)\right| \geq k$. This is a contradiction. Hence $L\left(x_{1}\right) \geq a$. Since $w$ is adjacent to $x_{1}$, this implies $L\left(x_{1}\right) \geq a+h \geq 2 h$.

Suppose that $L\left(x_{1}\right) \geq k+h$. We have $L\left(y_{1}\right)>a$ because $a<k$ and $\left|L\left(y_{1}\right)-a\right| \geq k$. Since $y_{1}$ and $w$ are at distance 2 in the tree, this implies $L\left(y_{1}\right) \geq a+k \geq h+k$. Consequently, adjacent vertices $x_{1}$ and $y_{1}$ have labels at least $k+h$. These labels must differ by at least $h$. Hence one of these labels is at least $k+2 h \geq 2 k$. The largest labeled sibling of the corresponding vertex must have label at least $(\Delta-2) k+2 k=\Delta k \geq(2 \Delta-1) h$. This is a contradiction. Hence $L\left(x_{1}\right)<k+h$.

Together with the previously proved inequality, this gives $2 h \leq L\left(x_{1}\right)<k+h$, which is part 1 . of Claim 2, for $r=1$.

Suppose that $L\left(y_{1}\right)<L\left(x_{1}\right)$. Since vertices $x_{1}$ and $y_{1}$ are adjacent, this implies $L\left(y_{1}\right) \leq L\left(x_{1}\right)-h<$ $k$. We also have $a<k$ and $L\left(y_{1}\right)-a \geq k$. This is a contradiction. Hence $L\left(y_{1}\right) \geq L\left(x_{1}\right)$. Since vertices $x_{1}$ and $y_{1}$ are adjacent, this implies $L\left(y_{1}\right) \geq L\left(x_{1}\right)+h \geq 3 h$.

Suppose that $L\left(y_{1}\right) \geq 2 k$. Then the largest labeled sibling of $y_{1}$ must have label at least $(\Delta-2) k+$ $2 k=\Delta k \geq(2 \Delta-1) h$. This is a contradiction. Hence $L\left(y_{1}\right)<2 k$.

Together with the previously proved inequality, this gives $3 h \leq L\left(y_{1}\right)<2 k$, which is part 2 . of Claim 2, for $r=1$. Hence Claim 2 is proved for $r=1$.

Now we prove the inductive step. Suppose that Claim 2 is true for $1,2, \ldots, r-1$. We prove it for $r$. Suppose that $L\left(x_{r}\right)<L\left(y_{r-1}\right)$. Hence $L\left(x_{r}\right)<r k$. We have $L\left(x_{r-1}\right)<(r-1) k+h<r k$. Vertex $x_{r}$ has $r-1$ siblings with smaller labels. Hence there are $r+1$ vertices (these $r-1$ siblings, $x_{r}$ itself, and $x_{r-1}$ ) which have labels differing by at most $k$, all smaller than $r k$. This is a contradiction. Hence $L\left(x_{r}\right) \geq L\left(y_{r-1}\right)$. Since $x_{r}$ and $y_{r-1}$ are adjacent, this implies $L\left(x_{r}\right) \geq L\left(y_{r-1}\right)+h \geq 2 r h$. Suppose that $L\left(x_{r}\right) \geq r k+h$. We have $L\left(y_{r}\right)>L\left(y_{r-1}\right)$. (Otherwise there would be $r+1$ vertices with labels differing by at most $k$, all smaller than $r k: r-1$ siblings of $y_{r}$ with smaller labels, $y_{r}$ itself, and $y_{r-1}$. This is impossible.) Since $y_{r}$ and $y_{r-1}$ are at distance 2 in the tree, this implies $L\left(y_{r}\right) \geq L\left(y_{r-1}\right)+k$. Hence $L\left(y_{r}\right) \geq(2 r-1) h+k \geq r k+h$. Both vertices $x_{r}$ and $y_{r}$ have labels at least $r k+h$. Since these vertices are adjacent, their labels must differ by at least $h$. Hence one of them is at least $r k+2 h \geq(r+1) k$. It has $\Delta-1-r$ siblings with larger labels. Hence its largest labeled sibling has label at least $(r+1) k+(\Delta-1-r) k=\Delta k \geq(2 \Delta-1) h$. This is a contradiction. Hence $L\left(x_{r}\right)<r k+h$.

Together with the previously proved inequality, this gives $2 r h \leq L\left(x_{r}\right)<r k+h$. This is part 1 . of the inductive step of Claim 2. 
Suppose that $L\left(y_{r}\right)<L\left(x_{r}\right)$. Since vertices $x_{r}$ and $y_{r}$ are adjacent, this implies $L\left(y_{r}\right) \leq L\left(x_{r}\right)-h<$ $r k$. Hence there are $r+1$ vertices with labels differing by at most $k$, all smaller than $r k: r-1$ siblings of $y_{r}$ with smaller labels, $y_{r}$ itself, and $y_{r-1}$. This is a contradiction. Hence $L\left(y_{r}\right) \geq L\left(x_{r}\right)$. Since $x_{r}$ and $y_{r}$ are adjacent, this implies $L\left(y_{r}\right) \geq L\left(x_{r}\right)+h \geq(2 r+1) h$.

Suppose that $L\left(y_{r}\right) \geq(r+1) k$. Vertex $y_{r}$ has $\Delta-1-r$ siblings with larger labels. Hence its largest labeled sibling has label at least $(r+1) k+(\Delta-1-r) k=\Delta k \geq(2 \Delta-1) h$. This is a contradiction. Hence $L\left(y_{r}\right)<(r+1) k$.

Together with the previously proved inequality this gives $(2 r+1) h \leq L\left(y_{r}\right)<(r+1) k$, which is part 2. of the inductive step of Claim 2. This conludes the proof of Claim 2.

Our theorem is now an immediate consequence of Claim 2, for $r=\Delta-1$ : we get $L\left(y_{\Delta-1}\right) \geq$ $(2(\Delta-1)+1) h=(2 \Delta-1) h$. This is a contradiction. Hence every $L(h, k)$-labeling of $T$ must have some label at least $(2 \Delta-1) h$.

Theorem 3.3 If $\frac{\Delta}{2 \Delta-1} k \leq h<k$ then $\lambda(\Delta, h, k)=\Delta k$.

Proof: In order to prove $\lambda(\Delta, h, k) \leq \Delta k$, consider any complete tree of degree $\Delta$, rooted at $r$. Consider the following labeling $L$. All labels are taken from the set $S=\{0, k, 2 k, \ldots, \Delta k\} . L(r)=0$. Children of $r$ get labels $k, 2 k, \ldots, \Delta k$. Children of any node $v \neq r$ get all labels from $S$, except that of $v$ and of the parent of $v$. Since $h<k$, labeling $L$ is an $L(h, k)$-labeling .

The proof of $\lambda(\Delta, h, k) \geq \Delta k$ is similar to that of the lower bound in Theorem 3.2, using the assumption $h \geq \frac{\Delta}{2 \Delta-1} k$.

\section{Minimum span for stars}

As we have seen in the proofs of lower bounds in Section 3 , relatively large values of $\lambda(\Delta, h, k)$ are witnessed by trees of fairly large height. In this section we show that this fact is not accidental. In particular, for trees of height 1 , i.e., for stars, the span of $L(h, k)$-labelings is smaller. More precisely, we prove the following result.

Theorem 4.1 For stars of degree $\Delta$, the minimum span of an $L(h, k)$-labeling is:

1. $(\Delta-1) k$, if $h<k / 2$;

2. $(\Delta-2) k+2 h$, if $k / 2 \leq h \leq k$; 
3. $(\Delta-1) k+h$, if $h>k$.

Proof: Case 1. $h<k / 2$.

Consider the following labeling of the star of degree $\Delta$. The center of the star gets label $\lfloor k / 2\rfloor$, and its leaves get labels $0, k, 2 k, \ldots,(\Delta-1) k$. Since $h \leq k / 2$, this is an $L(h, k)$-labeling . Its span is $(\Delta-1) k$. On the other hand, for any $L(h, k)$-labeling of the star, $\Delta$ values differing by at least $k$ are needed for the leaves, hence the largest label must be at least $(\Delta-1) k$.

Case 2. $k / 2 \leq h \leq k$.

Consider the following labeling of the star of degree $\Delta$. The center of the star gets label $h$, and its leaves get labels $0,2 h, 2 h+k, \ldots, 2 h+(\Delta-2) k$. Since $k / 2 \leq h$, this is an $L(h, k)$-labeling . Its span is $(\Delta-2) k+2 h$.

In order to prove the lower bound, consider any $L(h, k)$-labeling of the star of degree $\Delta$. If the center has label 0 then labels of leaves (in increasing order) must be at least $h, h+k, \ldots, h+(\Delta-1) k$. Since $h+(\Delta-1) k \geq(\Delta-2) k+2 h$, we are done. If 0 is the label of a leaf, then consider labels of leaves in increasing order. Since they differ by at least $k$, they must be of the form $0 \leq k+\alpha_{1}, \leq 2 k+\alpha_{2}, \ldots, \leq(\Delta-1) k+\alpha_{\Delta-1}$, where $0 \leq \alpha_{1} \leq \alpha_{2} \leq \cdots \leq \alpha_{\Delta-1}$. If the center has label larger than $(\Delta-1) k+\alpha_{\Delta-1}$, it is at least $h+(\Delta-1) k \geq(\Delta-2) k+2 h$, and we are done. Otherwise, let $i$ be the index for which the value $x$ of the center satisfies the inequalities $i k+\alpha_{i} \leq x \leq(i+1) k+\alpha_{i+1}$. Since $x$ must differ by at least $h$ from the label of each leaf, we have $(i+1) k+\alpha_{i+1}-\left(i k+\alpha_{i}\right) \geq 2 h$, hence $\alpha_{i+1} \geq \alpha_{i}+2 h-k$, which implies $\alpha_{\Delta-1} \geq 2 h-k$. Consequently, the span of the labeling is at least $(\Delta-1) k+2 h-k=(\Delta-2) k+2 h$.

Case 3. $h>k$.

Consider the following labeling of the star of degree $\Delta$. The center of the star gets label $(\Delta-1) k+h$ and its leaves get labels $0, k, 2 k, \ldots,(\Delta-1) k$. This is an $L(h, k)$-labeling, for any values of $h$ and $k$. Its span is $(\Delta-1) k+h$.

The proof of the lower bound is similar to that in Case 2, using the assumption $h \geq k$.

\section{Conclusion}

We established the optimal span of an $L(h, k)$-labeling for trees of maximum degree $\Delta$ and $h<k$. For $h \geq k$, values of optimal span follow from [13]. For trees of degree $\Delta$ and heigth 1, i.e. for stars, we obtained the exact values of optimal span for arbitrary parameters $h$ and $k$. These values are smaller than values of optimal span for the class of all trees of maximum degree $\Delta$.

An interesting open problem is to generalize the algorithmic result from [9] to the case of arbitrary 
$h$ and $k$ : find a polynomial algorithm (or prove that it does not exist) to determine the minimum span of an $L(h, k)$-labeling for an arbitrary tree. (Recall that such an algorithm was given in [9], for $h=2$ and $k=1$.)

Finally, it would be interesting to determine the minimum span of an $L(h, k)$-labeling for important classes of graphs, such as, e.g., hypercubes, and to find good bounds on this number for arbitrary graphs.

\section{References}

[1] K.I. Aardal, S.P.M. van Hoesel, A.M.C.A. Koster, C. Mannino and A. Sassano. Models and Solution Techniques for Frequency Assignment Problems. ZIB-Report 01-40, Konrad-ZuseZentrum fur Informationstechnik Berlin, 2001.

[2] A.A.Bertossi, C.Pinotti, R.B.Tan, $L(2,1)-$ and $L(2,1,1)$-labeling of graphs with applications to channel assignement in wireless networks, Proc. 4th International Workshop on Discrete Algorithms and Methods for Mobile Computing and Communications (DIAL-M 2000).

[3] H.L.Bodlaender, T.Kloks, R.B.Tan, J.Van Leeuwen, Lambda coloring of Graphs, Proc. 17th Ann. Symp. on Theoretical Aspects of Computer Science (STACS 2000), LNCS 1770, 395-406.

[4] T. Calamoneri, Exact Solution of a Class of Frequency Assignment Problems in Cellular Networks (Extended Abstract), 8th Italian Conference on Theoretical Computer Science (ICTCS'03), LNCS 2841, 163-173.

[5] T. Calamoneri and R. Petreschi, L(2,1)-Labeling of Planar Graphs (Extended Abstract), Proc. 5th ACM Int. Workshop on Discrete Algorithms and Methods for Mobile Computing and Communications (DIAL M '01), 28-33. Extended version accepted for publication in the Journal on Parallel and Distributed Computing.

[6] T. Calamoneri, R.Petreschi, Edge-clique graphs and the lambda-coloring problem, Journal of the Brazilian Computer Society, Special Issue in honor of Jaime Szwarcfiter's 60th birthday (2002).

[7] T.Calamoneri, R.Petreschi, L(2,1)-coloring matrogenic graphs (Extended Abstract), Latin American Theoretical Informatics (LATIN '02), LNCS 2286, 236-247.

[8] T. Calamoneri, and R. Petreschi, On the Radiocoloring Problem, Proc. 4th Int. Workshop on Distributed Computing, Mobile and Wireless Computing (IWDC'02), LNCS 2571, 118-127. 
[9] G.J. Chang and D. Kuo, The L(2,1)-labeling problem on graphs, SIAM J. Discr. Math. 9 (1996), 309-316.

[10] J.P. Georges and D.W. Mauro, Generalized vertex labelings with a condition at distance two, Congr. Numer. 109 (1995), 141-159.

[11] J.P. Georges and D.W. Mauro, On the size of graphs labeled with a condition at distance two, J. Graph Theory 22 (1996), 47-57.

[12] J.P. Georges and D.W. Mauro, Some results on $\lambda_{k}^{j}$-numbers of the products of complete graphs, Congr. Numer. 140 (1999), 141-160.

[13] J.P. Georges and D.W. Mauro, Labeling trees with a condition at distance two, Discrete Mat. 269 (2003), 127-148.

[14] J.P. Georges, D.W. Mauro and M.I. Stein, Labeling products of complete graphs with a condition at distance two, SIAM J. Discr. Math. 14 (2000), 28-35.

[15] J.R. Griggs and R.K.Yeh, Labeling graphs with a condition at distance 2, SIAM J. Discr. Math. 5 (1992), 586-595.

[16] W.K. Hale, Frequency assignment: Theory and application, Proc. IEEE 68 (1980), 1497-1514.

[17] J. van den Heuvel, R.A. Leese and M.A. Shepherd, Graph Labelling and Radio Channel Assignment, Journal of Graph Theory, 29 (1998), 263-283.

[18] T.R.Jensen and B.Toft, Graph coloring problems, John Wiley \& Sons, New York 1995.

[19] D. Liu and R.K.Yeh, On distance two labelings of graphs, Ars Combinatoria 47 (1997), 13-22.

[20] R.A. Murphey, P.M. Pardalos and M.G.C. Resende, Frequency Assignment Problems, in Handbook of Combinatorial Optimization, D.-Z. Du and P.M. Pardalos (Eds.) (1999), 295-377, Kluwer Academic Publishers.

[21] D. Sakai, Labeling chordal graphs: Distance 2 condition, SIAM J. Discr. Math. 7 (1994), 133-140.

[22] M. Shepherd, Radio Channel Assignment, Ph.D. thesis, Merton College, Oxford (1998).

[23] M.A. Whittlesey, J.P. Georges and D.W. Mauro, On the $\lambda$ number of $Q_{n}$ and related graphs, SIAM J. Discr. Math. 8 (1995), 499-506. 